\title{
NONLINEAR EVOLUTION EQUATIONS AND PRODUCT STABLE OPERATORS ON BANACH SPACES
}

\author{
BY \\ G. F. WEBB
}

\begin{abstract}
The method of product integration is used to obtain solutions to the time dependent Banach space differential equation $u^{\prime}(t)=A(t)(u(t)), t \geqq 0$, where $A$ is a function from $[0, \infty)$ to the set of nonlinear operators from the Banach space $X$ to itself and $u$ is a function from $[0, \infty)$ to $X$. The main requirements placed on $A$ are that $A$ is $m$-dissipative and product stable on its domain. Applications are given to a linear partial differential equation, to nonlinear dissipative operators in Hilbert space, and to continuous, $m$-dissipative, everywhere defined operators in Banach spaces.
\end{abstract}

1. Introduction. We consider the existence problem for the time dependent nonlinear evolution equation

$$
u^{\prime}(t)=A(t)(u(t)), \quad t \geqq 0,
$$

where $A$ is a mapping from $[0, \infty)$ to the set of (possibly nonlinear) operators on the Banach space $X$ and $u$ is a function from $[0, \infty)$ to $X$. The linear equation (1.1) has been treated by T. Kato [10], K. Yosida [20], J. Goldstein [8] as well as many others. More recently such authors as T. Kato [11], J. Dorroh [7], and R. Martin [13] have considered the nonlinear equation (1.1).

Previous authors have usually required a continuity condition on $A(t)$ as a function in $t$. The main objective of this paper is to treat the existence problem for (1.1) in cases for which such continuity conditions do not hold. We consider (1.1) in the form $u^{\prime}(t)=F^{\prime}(t) A(t) u(t)$ where $F$ is an absolutely continuous function from $[0, \infty)$ to the Banach algebra of bounded linear operators on $X$. The method used to construct solutions is product integration. In $\$ 2$ definitions are stated, in $\$ 3$ the main theorems are proved, and in $\$ 4$ examples and applications are given.

2. Definitions. Let $X$ be a Banach space, let $B(X)$ be the Banach algebra of bounded linear operators on $X$, and let $N(X)$ be the set of mappings (possibly nonlinear) from a subset of $X$ to $X$.

Definition 2.1. A function $F$ from $[0, \infty)$ to $B(X)$ is absolutely continuous provided that if $0 \leqq u \leqq v$ and $c>0$ there exists $d>0$ such that if $\left\{\left(s_{i}, t_{i}\right)\right\}_{i=0}^{n}$ is a sequence of disjoint intervals in $[u, v]$ and $\sum_{i=0}^{n}\left(s_{i}-t_{i}\right)<d$, then $\sum_{i=0}^{n}\left\|F\left(s_{i}\right)-F\left(t_{i}\right)\right\|$

Received by the editors June 1,1970 .

AMS 1969 subject classifications. Primary 3495, 3436; Secondary 3535, 3537.

Key words and phrases. Nonlinear evolution equation, product integral, $m$-dissipative mapping, product stable operator, evolution operator.

Copyright (C) 1971, American Mathematical Society 
$<c$. The greatest such number $d$ is the modulus of absolute continuity of $F$ on $[u, v]$ with respect to $c$.

We remark that if $F$ is an absolutely continuous function from $[0, \infty)$ to $B(X)$ and $0 \leqq u \leqq v$, then $F$ is of bounded variation on $[u, v]$, i.e., there is a number $N$ such that if $\left\{s_{i}\right\}_{i=0}^{n}$ is a chain from $u$ to $v$ then $\sum_{i=1}^{n}\left\|F\left(s_{i}\right)-F\left(s_{i-1}\right)\right\| \leqq N$ and the least such number $N$ is denoted by $\int_{u}^{v}\|d F\|$.

Definition 2.2. Let $A \in N(X)$ and let $F$ be a function from $[0, \infty)$ to $B(X)$. Define $A$ to be $m$-dissipative with respect to $F$ provided that if $0 \leqq s<t$ and $p, q \in \operatorname{domain} A$ then the following hold:

$$
\begin{gathered}
\|(I-(F(t)-F(s)) A) p-(I-(F(t)-F(s)) A) q\| \geqq\|p-q\|, \\
\quad \text { range }(I-(F(t)-F(s)) A)=X .
\end{gathered}
$$

Note that (2.1) and (2.2) imply that for $0 \leqq s<t,(I-(F(t)-F(s)) A)$ is one-to-one, $(I-(F(t)-F(s)) A)^{-1}$ is defined on all of $X$, and if $p, q \in X$, then

$$
\left\|(I-(F(t)-F(s)) A)^{-1} p-(I-(F(t)-F(s)) A)^{-1} q\right\| \leqq\|p-q\| .
$$

Definition 2.3. Let $F$ be a function from $[0, \infty)$ to $B(X)$ and let $A$ be a function from $[0, \infty)$ to $N(X)$ such that $A(t)$ is $m$-dissipative with respect to $F$ for $t \geqq 0$. If $0 \leqq u \leqq v$ and $s=\left\{s_{i}\right\}_{i=0}^{n}$ is a chain from $u$ to $v$, denote $\left(I-\left(F\left(s_{i}\right)-F\left(s_{i-1}\right)\right) A\left(s_{i-1}\right)\right)^{-1}$ by $\left[A, F, s_{i}\right]$ and denote $\prod_{i=1}^{n}\left[A, F, s_{i}\right]$ by $\prod_{s}[A, F]$ (where $\prod_{i=1}^{n}\left[A, F, s_{i}\right]$ $=\left[A, F, s_{n}\right]\left[A, F, s_{n-1}\right] \cdots\left[A, F, s_{1}\right]$ and the product operation is composition of mappings). Suppose that $p \in X, 0 \leqq u \leqq v$, and $z$ is a point of $X$ such that if $c>0$ there exists a chain $s$ from $u$ to $v$ such that if $t$ is a refinement of $s$ then $\left\|z-\prod_{t}[A, F] p\right\|<c$. Define $z$ to be the product integral of $A$ with respect to $F$ for $p$ from $u$ to $v$ and denote $z$ by $\prod_{u}^{v}[A, F] p$.

DefinItION 2.4. Let $0 \leqq u \leqq v$. A sequence of chains $\left\{s(1)_{i}\right\}_{i=0}^{r_{1}},\left\{s(2)_{i}\right\}_{i=0}^{\gamma_{2}}, \ldots$ from $u$ to $v$ is said to be admissible provided that if $n$ is a positive integer then the following hold:

(1) $s(n+1)$ is a refinement of $s(n)$,

(2) either $\max \left\{s(n)_{i}-s(n)_{i-1} \mid 1 \leqq i \leqq r_{n}\right\}<1 / n$ or if $s(n)_{j}-s(n)_{j-1}>1 / n$ for some integer $j$ in $\left[1, r_{n}\right]$, then for every integer $m>n$ there is no point $s(m)_{i}$ of $\left\{s(m)_{i}\right\}_{i=0}^{r_{m}}$ such that $s(n)_{j-1}<s(m)_{i}<s(n)_{j}$.

Definition 2.5. Let $A$ be a function from $[0, \infty)$ to $N(X)$, let $F$ be a function from $[0, \infty)$ to $B(X)$, and let $E \subseteq X$ such that (1) $A(t)$ is $m$-dissipative with respect to $F$ for $t \geqq 0$, (2) $E \subseteq$ domain $A(t)$ for $t \geqq 0$, and (3) $(I-(F(t)-F(s)) A(s))^{-1}(E) \subseteq E$ for $0 \leqq s<t$. We define $A$ to be product stable with respect to $F$ on $E$ provided that the following are true:

(2.4) If $p \in E$ and $0 \leqq v$, then there is a positive number $M(F, p, v)$ such that if $0 \leqq u \leqq x \leqq y \leqq v$ and $s$ is a chain from $u$ to $y$ then $\left\|A(x) \prod_{s}[A, F] p\right\| \leqq M(F, p, v)$.

(2.5) If $p \in E, 0 \leqq v$, and $c>0$, there exists $d>0$ such that if $0<y \leqq d, s$ is a chain from $v$ to $v+y$ and $v \leqq x \leqq v+y$, then $\left\|A(x) \prod_{s}[A, F] p-A(v) p\right\|<c$. 
(2.6) If $p \in E, 0 \leqq u \leqq v$, and $s(1), s(2), \ldots$ is an admissible sequence of chains from $u$ to $v$ such that $\lim _{n \rightarrow \infty} \prod_{s(n)}[A, F] p$ exists, then $\lim _{n \rightarrow \infty} \prod_{s(n)}[A, F] p \in E$.

3. An existence theorem. With the definitions above we state the following theorem:

TheOREM 3.1. Let $A$ be a function from $[0, \infty)$ to $N(X)$, let $F$ be an absolutely continuous function from $[0, \infty)$ to $B(X)$, and let $E$ be a subset of $X$ such that

(I) $A(t)$ is $m$-dissipative with respect to $F$ for $t \geqq 0$.

(II) $E \subseteq$ domain $A(t)$ for $t \geqq 0$.

(III) $E$ is invariant under $(I-(F(t)-F(s)) A(s))^{-1}$ for $0 \leqq s<t$, i.e.,

$$
(I-(F(t)-F(s)) A(s))^{-1}(E) \subseteq E .
$$

(IV) $A$ is product stable with respect to $F$ on $E$.

If $p \in E$ and $0 \leqq u \leqq v$ then $\prod_{u}^{v}[A, F] p$ exists and $\prod_{u}^{v}[A, F] p \in E$.

Examples satisfying (I)-(IV) are given in $\S 4$. Theorem 3.1 will be proved by means of a sequence of lemmas each of which is under the hypothesis of the theorem.

LEMMA 3.1. Let $p \in E$, let $0 \leqq u \leqq v$, let $\left\{s_{i}\right\}_{i=0}^{n}$ be a chain from $u$ to $v$, and let $M(F, p, v)$ be the positive number as in (2.4). If $1 \leqq j \leqq n$ and $z=\prod_{i=1}^{i-1}\left[A, F, s_{i}\right] p$, then

$$
\prod_{i=j}^{n}\left[A, F, s_{i}\right] z-z=\sum_{i=j}^{n}\left(F\left(s_{i}\right)-F\left(s_{i-1}\right)\right) A\left(s_{i-1}\right) \prod_{k=j}^{i}\left[A, F, s_{k}\right] z,
$$

and

$$
\left\|\prod_{i=j}^{n}\left[A, F, s_{i}\right] z-z\right\| \leqq M(F, p, v) \sum_{i=j}^{n}\left\|F\left(s_{i}\right)-F\left(s_{i-1}\right)\right\| .
$$

Proof. To establish (3.1) we see that

$$
\begin{aligned}
\prod_{i=j}^{n}\left[A, F, s_{i}\right] z-z & =\sum_{i=j}^{n}\left[\prod_{k=j}^{i}\left[A, F, s_{k}\right] z-\prod_{k=j}^{i-1}\left[A, F, s_{k}\right] z\right] \\
& =\sum_{i=j}^{n}\left(F\left(s_{i}\right)-F\left(s_{i-1}\right)\right) A\left(s_{i-1}\right) \prod_{k=j}^{i}\left[A, F, s_{k}\right] z .
\end{aligned}
$$

Then (3.2) follows immediately from (3.1) and (2.4).

We require now the following definitions: Let $u \geqq 0$ and define

$$
Q_{u}=\{K \subseteq[u, \infty) \mid u \in K, K \text { is bounded, every nonempty sub- }
$$
set of $K$ has a smallest number in $K$, and if $\left\{x_{i}\right\}_{i=1}^{\infty}$ is an infinite increasing sequence in $K$ then $\left.\lim \left\{x_{i}\right\}_{i=1}^{\infty} \notin K\right\}$.

If $K \in Q_{u}$ and $x>u$ let $K_{x}=\{y \in K \mid y<x\}$ and if $K \in Q_{u}$ let $K^{\prime}=\{x \mid x$ is the limit of an infinite increasing sequence in $K\}$. We remark that if $K \in Q_{u}$ then $K^{\prime}$ is closed and countable. 
Lemma 3.2. Let $u \geqq 0$, let $K \in Q_{u}$, and let $v=\sup K$. There is a sequence of chains $\left\{s(1)_{i}\right\}_{i=0}^{m_{1}},\left\{s(2)_{i}\right\}_{i=0}^{m_{2}}, \ldots$ from $u$ to $v$ such that for each positive integer $n$ :

(3.4) $s(n+1)$ is a refinement of $s(n)$.

(3.5) If $1 \leqq i \leqq m_{n}$, then $s(n)_{i} \in K \cup K^{\prime}$; if $s(n)_{i} \in K$, then $s(n)_{i}$ is the first point of $K$ which follows $s(n)_{i-1}$; if $s(n)_{i} \in K^{\prime}$, then $s(n)_{i-1} \in K$; and $\sum_{i, s(n)_{i} \in K^{\prime}}\left|s(n)_{i}-s(n)_{i-1}\right|$ $<\min \left\{b_{n}, 1 / n\right\}$ where $b_{n}$ is the modulus of absolute continuity of $F$ on $[u, v]$ with respect to $1 / 2^{n}$.

Moreover, if $p \in E$ there is a unique point $z$ of $E$ such that if $s(1), s(2), \ldots$ is any sequence of chains from $u$ to $v$ satisfying (3.4) and (3.5) then $\prod_{s(1)}[A, F] p$, $\prod_{s(2)}[A, F] p, \ldots$ converges to $z$.

Proof. The sequence of chains $s(1), s(2), \ldots$ satisfying (3.4) and (3.5) exists by virtue of the covering theorem and the fact that $K^{\prime}$ is closed. Suppose now that $\left\{s(1)_{i}\right\}_{i=0}^{m_{1}},\left\{s(2)_{i}\right\}_{i=0}^{m_{2}}, \ldots$ is any sequence of chains from $u$ to $v$ satisfying (3.4) and (3.5). Let $p \in E$, let $n$ be a positive integer and let $\left\{w_{i}\right\}_{i=0}^{m_{n}}$ be an increasing sequence such that $w_{0}=0, w_{m_{n}}=m_{n+1}$, and if $i$ is an integer in $\left[1, m_{n}\right]$ then $s(n)_{i}=s(n+1)_{w_{i}}$. If $i$ is an integer in $\left[1, m_{n}\right]$ let $K_{i}=\prod_{j=w_{i-1}+1}^{w_{i}}\left[A, F, s(n+1)_{j}\right]$ and let $J_{i}$ $=\prod_{j=1}^{i}\left[A, F, s(n)_{j}\right]$. Noting that if $s(n)_{i} \in K, K_{i} J_{i-1} p=J_{i} p$, we see that

$$
\begin{aligned}
\| \prod_{s(n+1)}[A, F] p- & \prod_{s(n)}[A, F] p \| \\
& =\left\|\sum_{i=1}^{m_{n}}\left[\prod_{j=i}^{m_{n}} K_{j} J_{i-1} p-\prod_{j=i+1}^{m_{n}} K_{j} J_{i} p\right]\right\| \\
& \leqq \sum_{i=1}^{m_{n}}\left\|K_{i} J_{i-1} p-J_{i} p\right\| \quad(\text { by }(2.3)) \\
& =\sum_{i, s(n)_{i} \in K^{\prime}}\left\|K_{i} J_{i-1} p-J_{i} p\right\| \\
& \leqq \sum_{i, s(n)_{i} \in K^{\prime}}\left\|K_{i} J_{i-1} p-J_{i-1} p\right\|+\left\|J_{i-1} p-\left[A, F, s(n)_{i}\right] J_{i-1} p\right\| \\
& \leqq \sum_{i, s(n)_{i} \in K^{\prime}}\left[\sum_{j=w_{i-1}+1}^{w_{i}}\left\|F\left(s(n+1)_{j}\right)-F\left(s(n+1)_{j-1}\right)\right\| M(F, p, v)\right. \\
& <\left(1 / 2^{n-1}\right) M(F, p, v) . \quad \text { (by (3.2)) }
\end{aligned}
$$

Thus, $\prod_{s(1)}[A, F] p, \Pi_{s(2)}[A, F] p, \ldots$ is a Cauchy sequence and so let $z$ denote its limit. We observe that $s(1), s(2), \ldots$ is an admissible sequence of chains from $u$ to $v$ (Definition 2.4) and so $z \in E$ by virtue of (2.6). To show that $z$ is unique suppose that each of $s(1), s(2), \ldots$ and $t(1), t(2), \ldots$ is a sequence of chains from $u$ to $v$ 
satisfying (3.4) and (3.5). We see that there is a sequence $s\left(n_{1}\right), t\left(n_{2}\right), s\left(n_{3}\right), t\left(n_{4}\right), \ldots$ satisfying (3.4) and (3.5) and so $\prod_{s(1)}[A, F] p, \prod_{s(2)}[A, F] p, \ldots$ and $\prod_{t(1)}[A, F] p$, $\prod_{t(2)}[A, F] p, \ldots$ must converge to $z$ and the lemma is proved.

We will need the following notation: Let the unique point $z$ of Lemma 3.2 be denoted by $z(K, p)$. Suppose now that $p \in E, u \geqq 0, K \in Q_{u}, v=\sup K$, and $c>0$. By (2.5) there exists $d>0$ such that if $0<y \leqq d, s$ is a chain from $v$ to $v+y$ and $v \leqq x \leqq v+y$, then $\left\|A(x) \prod_{s}[A, F] z(K, p)-A(v) z(K, p)\right\|<c$. Let $d(K, p, c)$ denote one such number $d$. If $p \in E, u \geqq 0$, and $c>0$ define

$$
A(p, u, c)=\left\{K \in Q_{u} \mid \text { if } q \neq u \in K \text { then } q=\sup K_{q}+d\left(K_{q}, p, c\right)\right\} .
$$

We remark that if $K \in A(p, u, c)$ and $q \neq u \in K$ then $K_{q} \in A(p, u, c)$. Further, if $K$ and $J$ are two members of $A(p, u, c)$, then there exists $q \in K$ such that $K_{q}=J$ or $q \in J$ such that $J_{q}=K$.

LeMmA 3.3. If $p \in E, 0 \leqq u \leqq v$, and $c>0$, then there exists $K \in A(p, u, c)$ such that $v \leqq \sup K$.

Proof. Assume that there exists no $K \in A(p, u, c)$ such that $\sup K \geqq v$. Let $J=\bigcup_{K \in A(p, u, c)} K$. We have then that $J$ is bounded and further $J \in A(p, u, c)$. But then $J \cup\{\sup J+d(J, p, c)\} \in A(p, u, c)$ which implies that $\sup J+d(J, p, c) \in J$ and so we have a contradiction.

Lemma 3.4. Suppose $p \in E, u \geqq 0$, and $K \in Q_{u}$. We have the following:

(i) If $q \in K, q \neq u$, and $v=\sup K_{q}$, then $(I-(F(q)-F(v)) A(v))^{-1} z\left(K_{q}, p\right)$ $=z\left(K_{q} \cup\{q\}, p\right)$.

(ii) If $x \in K, x \neq u, y \in K^{\prime}, x<y, w=z\left(K_{x} \cup\{x\}, p\right)$, and $J=K_{y}-K_{x}$, then $z\left(K_{y}, p\right)=z(J, w)$.

Proof. To show (i) suppose that $s(1), s(2), \ldots$ is a sequence of chains from $u$ to $v$ satisfying (3.4) and (3.5) of Lemma 3.2 for $K_{q}$. Then $s(1) \cup\{q\}, s(2) \cup\{q\}, \ldots$ is a sequence of chains from $u$ to $q$ satisfying (3.4) and (3.5) of Lemma 3.2 for $K_{q} \cup\{q\}$ and thus $\prod_{s(1) \cup\{q)}[A, F] p, \prod_{s(2) \cup\{q\}}[A, F] p, \ldots$ converges to $z\left(K_{q} \cup\{q\}, p\right)$. Further, $\prod_{s(1)}[A, F] p, \prod_{s(2)}[A, F] p, \ldots$ converges to $z\left(K_{q}, p\right)$ and so by the continuity of $(I-(F(q)-F(v)) A(v))^{-1}$ (see (2.3)) we have (i).

To show (ii) suppose that $s(1), s(2), \ldots$ is a sequence of chains from $u$ to $x$ satisfying (3.4) and (3.5) of Lemma 3.2 for $K_{x} \cup\{x\}$. We note that $J \in Q_{x}$ and so let $t(1), t(2), \ldots$ be a sequence of chains from $x$ to $y$ satisfying (3.4) and (3.5) of Lemma 3.2 for $J$. Define $r(n)=s(n) \cup t(n)$ for each positive integer $n$. Then a subsequence $r\left(n_{1}\right), r\left(n_{2}\right), \ldots$ of $r(1), r(2), \ldots$ satisfies (3.4) and (3.5) of Lemma 3.2 for $K_{y}$. Thus $\prod_{r\left(n_{1}\right)}[A, F] p, \prod_{r\left(n_{2}\right)}[A, F] p, \ldots$ converges to $z\left(K_{y}, p\right), \prod_{s\left(n_{1}\right)}[A, F] p$, $\prod_{s\left(n_{2}\right)}[A, F] p, \ldots$ converges to $w, \prod_{t\left(n_{1}\right)}[A, F] w, \prod_{t\left(n_{2}\right)}[A, F] w, \ldots$ converges to $z(J, w)$, and hence $z\left(K_{y}, p\right)=z(J, w)$. 
LEMMA 3.5. Suppose $p \in E, 0 \leqq u \leqq v$, and $c>0$. There is a chain srom $u$ to $v$ such that if $t$ is a refinement of $s$ then $\left\|\Pi_{s}[A, F] p-\Pi_{t}[A, F] p\right\|<c$.

Proof. By Lemma 3.3 one of the following two cases must hold:

Case 1. There exists $K \in A(p, u, c)$ such that $v=\sup K$.

Case 2. There exists $K \in A(p, u, c)$ such that $v<\sup K$ and there exists no $q \in K$ such that $v<q<\sup K$.

We first consider Case 1. Let $s=\left\{s_{i}\right\}_{i=0}^{m}$ be a chain from $u$ to $v=\sup K$ such that if $i$ is an integer in $[1, m]$ then $s$ has the properties (a) if $s_{i} \in K$ then $s_{i}$ is the first point of $K$ which follows $s_{i-1}$; (b) if $s_{i} \notin K$ then $s_{i} \in K^{\prime}$ and $s_{i-1} \in K$; and (c) $\sum_{i, s_{i} \in K^{\prime}}\left|s_{i}-s_{i-1}\right|<b$ where $b$ is the modulus of absolute continuity of $F$ on $[u, v]$ with respect to $c$. Define $\left\{q_{i}\right\}_{i=0}^{m}$ as follows:

$$
\begin{aligned}
& q_{0}=p, \\
& q_{i}=z\left(K_{s_{i}} \cup\left\{s_{i}\right\}, p\right) \quad \text { if } s_{i} \in K, \\
& q_{i}=z\left(K_{s_{i}}, p\right) \quad \text { if } s_{i} \in K^{\prime} .
\end{aligned}
$$

We observe that if $s_{i} \in K, i \neq 0$, then by Lemma 3.4(i) and (3.6) we have the following:

$$
\begin{aligned}
q_{i} & =z\left(K_{s_{i}} \cup\left\{s_{i}\right\}, p\right) \\
& =\left(I-\left(F\left(\sup K_{s_{i}}+d\left(K_{s_{i}}, p, c\right)\right)-F\left(\sup K_{s_{i}}\right)\right) A\left(\sup K_{s_{i}}\right)\right)^{-1} z\left(K_{s_{i}}, p\right) \\
& =\left(I-\left(F\left(s_{i}\right)-F\left(s_{i-1}\right)\right) A\left(s_{i-1}\right)\right)^{-1} q_{i-1} .
\end{aligned}
$$

Further, if $s_{i} \in K, i \neq 0, r$ is a chain from $s_{i-1}$ to $y$ where $s_{i-1} \leqq y \leqq s_{i}$, and $x \in\left[s_{i-1}, y\right]$, then by (3.6) we have that

$$
\left\|A(x) \prod_{r}[A, F] q_{i-1}-A\left(s_{i-1}\right) q_{i-1}\right\|<c .
$$

If $s_{i} \in K^{\prime}$ we have by Lemma 3.4(ii) that

$$
q_{i}=z\left(K_{s_{i}}, p\right)=z\left(K_{s_{i}}-K_{s_{i-1}}, q_{i-1}\right) \text {. }
$$

Further, by using Lemma 3.1 and (c) above we have that

$$
\sum_{i, s_{i} \in K^{\prime}}\left\|\prod_{r(i)}[A, F] q_{i-1}-q_{i-1}\right\| \leqq c M(F, p, v)
$$

where $r(i)$ is a chain from $s_{i-1}$ to $s_{i}$, and

$$
\begin{aligned}
\sum_{i, s_{i} \in K^{\prime}}\left\|q_{i}-q_{i-1}\right\| & =\sum_{i, s_{i} \in K^{\prime}}\left\|z\left(K_{s_{i}}-K_{s_{i-1}}, q_{i-1}\right)-q_{i-1}\right\| \\
& \leqq c M(F, p, v) .
\end{aligned}
$$

Suppose now that $t=\left\{t_{j}\right\}_{j=0}^{n}$ is a refinement of $s=\left\{s_{i}\right\}_{i=0}^{m}$, i.e., there is an increasing sequence $\left\{w_{i}\right\}_{i=0}^{m}$ such that $w_{0}=0, w_{m}=n$, and if $1 \leqq i \leqq m$ then $s_{i}=t_{w_{i}}$. Define 
$K_{i}=\prod_{j=w_{i-1}+1}^{w_{i}}\left[A, F, t_{j}\right]$ for $1 \leqq i \leqq m$. Then, using (3.8), (3.9), and (3.1) we see that

$$
\begin{aligned}
& \sum_{i, s_{i} \in K}\left\|K_{i} q_{i-1}-q_{i}\right\|=\sum_{i, s_{i} \in K}\left\|K_{i} q_{i-1}-\left[A, F, s_{i}\right] q_{i-1}\right\| \quad \text { (by (3.8)) } \\
& \leqq \sum_{i, s_{i} \in K}\left\|\left(I-\left(F\left(s_{i}\right)-F\left(s_{i-1}\right)\right) A\left(s_{i-1}\right)\right) K_{i} q_{i-1}-q_{i-1}\right\| \quad \text { (by (2.3)) } \\
& =\sum_{i, s_{i} \in K} \|\left[\prod_{j=w_{i}-1+1}^{w_{i}}\left[A, F, t_{j}\right] q_{i-1}-q_{i-1}\right] \\
& -\left(F\left(s_{i}\right)-F\left(s_{i-1}\right)\right) A\left(s_{i-1}\right) K_{i} q_{i-1} \| \\
& =\sum_{i, s_{i} \in K} \| \sum_{j=w_{i}-1+1}^{w_{i}}\left(F\left(t_{j}\right)-F\left(t_{j-1}\right)\right) \\
& \times\left(A\left(t_{j-1}\right) \prod_{k=w_{i-1}+1}^{j}\left[A, F, t_{k}\right] q_{i-1}-A\left(s_{i-1}\right) K_{i} q_{i-1}\right) \| \\
& \text { (by (3.1)) } \\
& \leqq \sum_{i, s_{i} \in K} \sum_{j=w_{i}-1+1}^{w_{i}}\left\|F\left(t_{j}\right)-F\left(t_{j-1}\right)\right\| \\
& \times\left(\left\|A\left(t_{j-1}\right) \prod_{k=w_{i-1}+1}^{j}\left[A, F, t_{k}\right] q_{i-1}-A\left(s_{i-1}\right) q_{i-1}\right\|\right. \\
& \left.+\left\|A\left(s_{i-1}\right) q_{i-1}-A\left(s_{i-1}\right) \prod_{k=w_{i-1}+1}^{w_{i}}\left[A, F, t_{k}\right] q_{i-1}\right\|\right) \\
& <2 c \int_{u}^{v}\|d F\| \quad \text { (by (3.9)) }
\end{aligned}
$$

Moreover, using (3.10), (3.11), and (3.12), we have that

$$
\begin{aligned}
\sum_{i, s_{i} \in K^{\prime}}\left\|K_{i} q_{i-1}-q_{i}\right\| & \leqq \sum_{i, s_{i} \in K^{\prime}}\left\|K_{i} q_{i-1}-q_{i-1}\right\|+\left\|q_{i-1}-z\left(K_{s_{i}}-K_{s_{i-1}}, q_{i-1}\right)\right\| \\
& <2 c M(F, p, v) .
\end{aligned}
$$

Thus,

$$
\begin{aligned}
\left\|\prod_{t}[A, F] p-q_{m}\right\| & =\left\|\sum_{i=1}^{m}\left[\prod_{j=i}^{m} K_{j} q_{i-1}-\prod_{j=i+1}^{m} K_{j} q_{i}\right]\right\| \\
& \leqq \sum_{i=1}^{m}\left\|K_{i} q_{i-1}-q_{i}\right\| \\
& =\sum_{i, s_{i} \in K}\left\|K_{i} q_{i-1}-q_{i}\right\|+\sum_{i, s_{i} \in K^{\prime}}\left\|K_{i} q_{i-1}-q_{i}\right\| \\
& <2 c \int_{u}^{v}\|d F\|+2 c M(F, p, v)
\end{aligned}
$$

and noting that $s$ is a refinement of itself we have the lemma established for Case 1 
Suppose now that Case 2 holds and Case 1 does not hold. Then, $\sup K \in K$, $K_{\text {sup } K} \in A(p, u, c)$, and $\sup \left(K_{\sup K}\right)<v$. Let $q=z\left(K_{\text {sup } K}, p\right)$ and let $w=\sup \left(K_{\sup K}\right)$ and note that by (3.6) sup $K=w+d\left(K_{\operatorname{sup~} K}, p, c\right)$. Further, let

$$
r=(I-(F(v)-F(w)) A(w))^{-1} q .
$$

In Case 1 (see (3.13)) we showed that there is a chain $\left\{s_{i}\right\}_{i=0}^{m}$ from $u$ to $w$ such that if $t$ is a refinement of $s$ then

$$
\left\|\prod_{t}[A, F] p-q\right\|<2 c\left(\int_{u}^{w}\|d F\|+M(F, p, w)\right) .
$$

Define $\left\{s_{i}^{\prime}\right\}_{i=0}^{m+1}$ by $s_{i}^{\prime}=s_{i}$ if $0 \leqq i \leqq m$ and $s_{m+1}^{\prime}=v$. Let $\left\{t_{i}\right\}_{i=0}^{n}$ be a refinement of $s^{\prime}$ and let $t_{k}=w$. We have then that

$$
\begin{aligned}
\left\|\prod_{t}[A, F] p-r\right\| \leqq & \prod_{i=k+1}^{n}\left[A, F, t_{i}\right] \prod_{j=1}^{k}\left[A, F, t_{j}\right] p-\prod_{i=k+1}^{n}\left[A, F, t_{i}\right] q \| \\
& +\left\|\prod_{i=k+1}^{n}\left[A, F, t_{i}\right] q-(I-(F(v)-F(w)) A(w))^{-1} q\right\| \\
< & 2 c\left(\int_{u}^{w}\|d F\|+M(F, p, w)\right) \\
& +\left\|(I-(F(v)-F(w)) A(w)) \prod_{i=k+1}^{n}\left[A, F, t_{i}\right] q-q\right\| \\
\leqq & 2 c\left(\int_{u}^{w}\|d F\|+M(F, p, w)\right)+\sum_{i=k+1}^{n}\left\|F\left(t_{i}\right)-F\left(t_{i-1}\right)\right\| \\
& \times\left(\left\|A\left(t_{i-1}\right) \prod_{j=k+1}^{i}\left[A, F, t_{j}\right] q-A(w) q\right\|\right. \\
& \quad 2 c\left(\int_{u}^{w}\|d F\|+M(F, p, w)\right)+2 c \int_{w}^{v}\|d F\|
\end{aligned}
$$

Thus, observing that $s$ is a refinement of itself we have the lemma established for Case 2.

We see then that if $p \in E$ and $0 \leqq u \leqq v$ the product integral $\prod_{u}^{v}[A, F] p$ of $A$ with respect to $F$ for $p$ from $u$ to $v$ exists (Definition 2.3) by virtue of Lemma 3.5 and the completeness of $X$. Further, it follows from (2.6) that $\prod_{u}^{v}[A, F] p \in E$. Hence, Theorem 3.1 is established. In [18] the author proved Theorem 3.1 in the special case that $A(t)=A$ is independent of $t, F(t)=t I$ for $t \geqq 0$, and $A$ is everywhere defined and continuous on $X$. In Example 4.3 we will consider a problem similar to this one in the time dependent case. Other authors, such as H. Brezis and 
A. Pazy [3], J. Mermin [14], and J. Neuberger [15] have obtained results similar to Theorem 3.1. Recently, M. Crandall and T. Liggett announced in [4] the following very general result in the time independent case: If $A(t)=A$ is $m$-dissipative with respect to $F(t)=t I, t \geqq 0$, and $p$ is in the domain of $A$, then $\lim _{n \rightarrow \infty}(I-(x / n) A)^{-n}$ exists for all $x \geqq 0$.

By virtue of Theorem 3.1 we state now the following definition:

Definition 3.1. Let $A$ be a function from $[0, \infty)$ to $N(X)$, let $F$ be an absolutely continuous function from $[0, \infty)$ to $B(X)$, and let $E$ be a subset of $X$ such that (I), (II), (III), and (IV) of Theorem 3.1 hold. If $0 \leqq u \leqq v$ define the mapping $U(v, u)$ from $E$ to $E$ by $U(v, u) p=\prod_{u}^{v}[A, F] p$ for each point $p \in E$ and call $U$ the evolution operator of $A$ with respect to $F$ on $E$.

One easily verifies that $U$ has the following properties:

(3.15) $U(u, u) p=p$ for $p \in E$ and $u \geqq 0$.

(3.16) $\|U(v, u) p-U(v, u) q\| \leqq\|p-q\|$ for $p, q \in E$ and $0 \leqq u \leqq v$ (by Lemma 3.5 and (2.3)).

(3.17) $U(v, w) U(w, u) p=U(v, u) p$ for $p \in E$ and $0 \leqq u \leqq w \leqq v$ (by Lemma 3.5).

(3.18) $\|U(v, u) p-p\| \leqq M(F, p, v) \int_{u}^{v}\|d F\|$ for $p \in E$ and $0 \leqq u \leqq v$ (by Lemma 3.1).

We remark further that if $0 \leqq u \leqq v$, then $U(v, u)$ can be extended by continuity ((3.16) above) to the closure $\bar{E}$ of $E$.

In the discussion that follows we require that the function $F$ from $[0, \infty)$ to $B(X)$ can be represented as the indefinite Bochner integral of its derivative. For a treatment of Bochner integration the reader is referred to [9]. We will use the following facts:

(3.19) If $F$ is absolutely continuous and has a strong derivative $F^{\prime}$ almost everywhere, then $F$ can be expressed as the indefinite Bochner integral of $F^{\prime}$ (see [9, Theorem 3.8.6, p. 88]).

(3.20) If $F^{\prime}$ is Bochner integrable then $\left\|F^{\prime}\right\|$ is Lebesgue integrable and $\lim _{h \rightarrow 0}(1 / h) \int_{t}^{t+h}\left\|F^{\prime}\right\|=\left\|F^{\prime}(t)\right\|$ for almost all $t \geqq 0$ (see [9, Theorems 3.7.4 and 3.8.5]).

TheOREM 3.2. Let $A$ be a function from $[0, \infty)$ to $N(X)$, let $F$ be an absolutely continuous function from $[0, \infty)$ to $B(X)$, and let $E \subseteq X$ such that (I), (II), (III), and (IV) of Theorem 3.1 are satisfied, and let $U$ be the evolution operator of $A$ with respect to $F$ on $E$. Further, let $F$ have a strong derivative almost everywhere on $[0, \infty)$. If $p \in E$ and $0 \leqq u$ then for almost all $t \geqq u$

$$
d^{+} / d t U(t, u) p=F^{+}(t) A(t) U(t, u) p
$$

Proof. Let $p \in E, 0 \leqq u, c>0$ and $t \geqq u$ such that $F^{\prime+}(t)$ exists and

$$
\lim _{h \rightarrow 0^{+}} 1 / h \int_{t}^{t+h}\left\|F^{\prime}\right\|=\left\|F^{\prime+}(t)\right\| .
$$


There exists $d>0$ such that if $0<h<d$ then

$$
\begin{gathered}
\left\|(1 / h)[F(t+h)-F(t)]-F^{\prime+}(t)\right\|<c, \\
\frac{1}{h} \int_{t}^{t+h}\left\|F^{\prime}\right\|<\left\|F^{\prime+}(t)\right\|+1,
\end{gathered}
$$

and if $0<h<d, s$ is a chain from $t$ to $t+h$ and $x \in[t, t+h]$ then

$$
\left\|A(x) \prod_{s}[A, F] U(t, u) p-A(t) U(t, u) p\right\|<c
$$

Let $0<h<d$. There is a chain $r=\left\{r_{i}\right\}_{i=0}^{n}$ from $t$ to $t+h$ such that

$$
\left\|\prod_{r}[A, F] U(t, u) p-U(t+h, t) U(t, u) p\right\|<c h .
$$

Then

$$
\begin{aligned}
&\left\|(1 / h)[U(t+h, u) p-U(t, u) p]-F^{\prime}(t) A(t) U(t, u) p\right\| \\
&<c+\frac{1}{h}\left\|\left[\prod_{r}[A, F] U(t, u) p-U(t, u) p\right]-[F(t+h)-F(t)] A(t) U(t, u) p\right\| \\
& \quad+\left\|(1 / h)[F(t+h)-F(t)] A(t) U(t, u) p-F^{\prime+}(t) A(t) U(t, u) p\right\| \\
& \leqq c+\frac{1}{h} \| \sum_{i=1}^{n}\left[F\left(r_{i}\right)-F\left(r_{i-1}\right)\right] \\
&+\left\|(1 / h)[F(t+h)-F(t)]-F^{\prime}+(t)\right\|\|A(t) U(t, u) p\| \\
& \leqq c+\frac{1}{h} \sum_{i=1}^{n}\left\|F\left(r_{i}\right)-F\left(r_{i-1}\right)\right\| c+c\|A(t) U(t, u) p\| \\
&= c+\frac{1}{h} \sum_{i=1}^{n}\left\|\int_{r_{i-1}}^{r_{i}} F^{\prime}\right\| c+c\|A(t) U(t, u) p\| \\
& \leqq c\left(1+\frac{1}{h} \sum_{i=1}^{n} \int_{r_{i}-1}^{r_{i}}\left\|F^{\prime}\right\|+\|A(t) U(t, u) p\|\right) \\
&= c\left(1+\frac{1}{h} \int_{t}^{t+h}\left\|F^{\prime}\right\|+\|A(t) U(t, u) p\|\right) \\
&< c\left(1+\left\|F^{\prime+}(t)\right\|+1+\|A(t) U(t, u) p\|\right)
\end{aligned}
$$

Thus, $d^{+} / d t U(t, u) p=F^{\prime+}(t) A(t) U(t, u) p$ and so the theorem is proved.

We conclude this section with the following theorem:

THEOREM 3.3. Let $A$ be a mapping from $[0, \infty)$ to $N(X)$, let each of $F$ and $G$ be an absolutely continuous function from $[0, \infty)$ to $B(X)$, and let $E$ be a subset of $X$ such 
that (I), (II), (III), and (IV) of Theorem 3.1 are satisfied for both $F$ and $G$ on $E$. Let $U_{F}$ and $U_{G}$ be the evolution operators of $A$ with respect to $F$ and $G$, respectively. If $p \in E$ and $0 \leqq u \leqq v$, then

$$
\left\|U_{F}(v, u) p-U_{G}(v, u) p\right\| \leqq M(F, p, v) \int_{u}^{v}\|d(F-G)\|
$$

and if each of $F$ and $G$ can be represented as the indefinite Bochner integral of $F^{\prime}$ and $G^{\prime}$, respectively, then

$$
\left\|U_{F}(v, u) p-U_{G}(v, u) p\right\| \leqq M(F, p, v) \int_{u}^{v}\left\|F^{\prime}-G^{\prime}\right\|
$$

Proof. Let $p \in E$, let $0 \leqq u \leqq v$, let $s=\left\{s_{i}\right\}_{i=0}^{n}$ be a chain from $u$ to $v$, let $q_{i}=\prod_{j=1}^{i-1}\left[A, F, s_{j}\right] p$ for each integer in $[2, n]$ and let $q_{1}=p$. Then,

$$
\begin{aligned}
& \left\|\prod_{s}[A, F] p-\prod_{s}[A, G] p\right\| \\
& =\left\|\sum_{i=1}^{n}\left[\prod_{j=i}^{n}\left[A, G, s_{j}\right] \prod_{j=1}^{i}\left[A, F, s_{j}\right] p-\prod_{j=i}^{n}\left[A, G, s_{j}\right] \prod_{j=1}^{i-1}\left[A, F, s_{j}\right] p\right]\right\| \\
& \leqq \sum_{i=1}^{n}\left\|\left[A, F, s_{i}\right] q_{i}-\left[A, G, s_{i}\right] q_{i}\right\| \\
& \leqq \sum_{i=1}^{n}\left\|\left(I-\left(G\left(s_{i}\right)-G\left(s_{i-1}\right)\right) A\left(s_{i-1}\right)\right)\left[A, F, s_{i}\right] q_{i}-q_{i}\right\| \\
& =\sum_{i=1}^{n}\left\|\left(\left(F\left(s_{i}\right)-F\left(s_{i-1}\right)\right)-\left(G\left(s_{i}\right)-G\left(s_{i-1}\right)\right)\right) A\left(s_{i-1}\right)\left[A, F, s_{i}\right] q_{i}\right\| \\
& \leqq \sum_{i=1}^{n}\left\|\left(F\left(s_{i}\right)-F\left(s_{i-1}\right)\right)-\left(G\left(s_{i}\right)-G\left(s_{i-1}\right)\right)\right\| M(F, p, v) \\
& \leqq M(F, p, v) \int_{u}^{v}\|d(F-G)\| .
\end{aligned}
$$

Thus, by Lemma 3.5 we have (3.25). Moreover, if $F$ and $G$ can be represented as the indefinite Bochner integral of $F^{\prime}$ and $G^{\prime}$, respectively, then from (3.27) we have that

$$
\begin{aligned}
\left\|\prod_{s}[A, F] p-\prod_{s}[A, G] p\right\| & \leqq \sum_{i=1}^{n}\left\|\left(F\left(s_{i}\right)-F\left(s_{i-1}\right)\right)-\left(G\left(s_{i}\right)-G\left(s_{i-1}\right)\right)\right\| M(F, p, v) \\
& \leqq M(F, p, v) \int_{u}^{v}\left\|F^{\prime}-G^{\prime}\right\| .
\end{aligned}
$$

Again, using Lemma 3.5 we have (3.26) and so the theorem is proved.

4. Examples. We first prove a theorem for the linear case that will be applied to a linear partial differential equation. 
THEOREM 4.1. Let $A$ be a closed, one-to-one, onto, linear operator defined on $D \subseteq X$ to $X$ where $X$ is a Banach space. Further, let $A x=\sum_{i=0}^{n} D_{i} x$ for $x \in D$, where, for each integer $i$ in $[0, n], D_{i}$ is a closable linear operator on $X$ and the domain of $D_{i}$ contains $D$. For each integer $i$ in $[0, n]$ let $F_{i}$ be an absolutely continuous function from $[0, \infty)$ to $B(X)$ and define $A(t)=A$ and $F(t)=\sum_{i=0}^{n} F_{i}(t) D_{i} A^{-1}$ for all $t \geqq 0$. Let $E=\bigcap_{n=1}^{\infty}$ domain $A^{n}$ and require that the following are satisfied:

(4.1) $\|(I-(F(t)-F(s)) A) p\| \geqq\|p\|$ for $p \in D, 0 \leqq s<t$,

(4.2) range $(I-(F(t)-F(s)) A)=X$ for $0 \leqq s<t$,

(4.3) $(I-(F(t)-F(s)) A)^{-1} A p=A(I-(F(t)-F(s)) A)^{-1} p$ for $p \in E, 0 \leqq s<t$.

Then, $F$ is an absolutely continuous function from $[0, \infty)$ to $B(X)$ and conditions (I), (II), (III), and (IV) of Theorem 3.1 are satisfied.

Proof. We first show that $F$ is absolutely continuous. Since $A^{-1}$ is closed and everywhere defined, we have that $A^{-1}$ is bounded by the closed graph theorem. For each integer $i$ in $[0, n]$ there is a closed extension $\bar{D}_{i}$ of $D_{i}$. Then, $\bar{D}_{i} A^{-1}$ is closed, everywhere defined and thus bounded by the closed graph theorem. Moreover, $\bar{D}_{i} A^{-1}=D_{i} A^{-1}$ and so $F(t) \in B(X)$ for $t \geqq 0$. If $0 \leqq s \leqq t$ then

$$
\|F(t)-F(s)\| \leqq \sum_{i=0}^{n}\left\|F_{i}(t)-F_{i}(s)\right\|\left\|D_{i} A^{-1}\right\|
$$

and so $F$ is absolutely continuous. We see that (I) is satisfied as a consequence of (4.1) and (4.2), (II) is satisfied since $A(t)=A$ for all $t \geqq 0$, and (III) is satisfied as a consequence of (4.3). To show that (IV) is satisfied we first see that if $p \in E$, $0 \leqq u<v$, and $s=\left\{s_{i}\right\}_{i=0}^{m}$ is a chain from $u$ to $v$, then

$$
\begin{aligned}
\| A \prod_{i=1}^{m}( & \left.I-\left(F\left(s_{i}\right)-F\left(s_{i-1}\right)\right) A\right)^{-1} p-A p \| \\
= & \left\|\prod_{i=1}^{m}\left(I-\left(F\left(s_{i}\right)-F\left(s_{i-1}\right)\right) A\right)^{-1} A p-A p\right\| \quad(\text { by }(4.3)) \\
& =\| \sum_{i=1}^{m}\left(\prod_{j=i}^{m}\left(I-\left(F\left(s_{j}\right)-F\left(s_{j-1}\right)\right) A\right)^{-1} A p\right. \\
& \leqq \sum_{i=1}^{m}\left\|\left(I-\left(F\left(s_{i}\right)-F\left(s_{i-1}\right)\right) A\right)^{-1} A p-A p\right\| \quad\left(\prod_{j=i+1}^{m}\left(I-\left(F\left(s_{j}\right)-F\left(s_{j-1}\right)\right) A\right)^{-1} A p\right) \| \\
& \leqq \sum_{i=1}^{m}\left\|A p-\left(I-\left(F\left(s_{i}\right)-F\left(s_{i-1}\right)\right) A\right) A p\right\| \\
& \leqq \sum_{i=1}^{m}\left\|F\left(s_{i}\right)-F\left(s_{i-1}\right)\right\|\left\|A^{2} p\right\| .
\end{aligned}
$$


Thus, we have (2.4) and (2.5) by the absolute continuity of $F$. To show (2.6) suppose that $p \in E, 0 \leqq u<v$ and $\left\{s(1)_{i}\right\}_{i=0}^{r_{1}},\left\{s(2)_{i}\right\}_{i=0}^{r_{2}}, \ldots$ is an admissible sequence of chains from $u$ to $v$ such that $\lim _{n \rightarrow \infty} \prod_{s(n)}[A, F] p$ exists. We will use (4.3) and (4.4) to show that if $q$ is a positive integer then $\lim _{n \rightarrow \infty} \prod_{s(n)}[A, F] A^{q} p$ exists. Suppose $m, n$ are positive integers such that $m>n$ and let $w$ be an increasing sequence such that $w_{0}=0, w_{r_{n}}=r_{m}$, and if $i$ is an integer in $\left[0, r_{n}\right]$ then $s(n)_{i}=s(m)_{w_{i}}$. If $i$ is an integer in $\left[1, r_{n}\right]$ let $K_{i}=\prod_{j=w_{i-1}+1}^{w_{i}}\left[A, F, s(m)_{j}\right]$ and let $J_{i}=\prod_{j=1}^{i}\left[A, F, s(n)_{j}\right]$. Further, let $L=\left\{i \in\left[1, r_{n}\right] \mid w_{i-1}+1 \neq w_{i}\right\}$. Then, if $q$ is a positive integer,

$$
\begin{aligned}
& \left\|\prod_{i=1}^{r_{m}}\left[A, F, s(m)_{i}\right] A^{q} p-\prod_{i=1}^{r_{n}}\left[A, F, s(n)_{i}\right] A^{q} p\right\| \\
& =\left\|\prod_{i=1}^{r_{n}} K_{i} A^{q} p-J_{r_{n}} A^{q} p\right\| \\
& =\left\|\sum_{i=1}^{r_{n}}\left[\prod_{j=i}^{r_{n}} K_{j} J_{i-1} A^{q} p-\prod_{j=i+1}^{r_{n}} K_{j} J_{i} A^{q} p\right]\right\| \\
& \leqq \sum_{i=1}^{r_{n}}\left\|K_{i} J_{i-1} A^{q} p-J_{i} A^{q} p\right\| \\
& =\sum_{i \in L}\left\|K_{i} J_{i-1} A^{q} p-\left[A, F, s(n)_{i}\right] J_{i-1} A^{q} p\right\| \\
& \leqq \sum_{i \in L}\left\|\left(I-\left(F\left(s(n)_{i}\right)-F\left(s(n)_{i-1}\right)\right) A\right) K_{i} J_{i-1} A^{q} p-J_{i-1} A^{q} p\right\| \\
& =\sum_{i \in L} \|\left[\prod_{j=w_{i-1}+1}^{w_{i}}\left[A, F, s(m)_{j}\right] J_{i-1} A^{q} p-J_{i-1} A^{q} p\right] \\
& -\left(F\left(s(n)_{i}\right)-F\left(s(n)_{i-1}\right)\right) A K_{i} J_{i-1} A^{q} p \| \\
& =\sum_{i \in L} \| \sum_{j=w_{i-1}+1}^{w_{i}}\left[F\left(s(m)_{j}\right)-F\left(s(m)_{j-1}\right)\right] \\
& \times\left[A \prod_{k=w_{i-1}+1}^{j}\left[A, F, s(m)_{k}\right] J_{i-1} A^{q} p-K_{i} A J_{i-1} A^{q} p\right] \| \\
& \leqq \sum_{i \in L} \sum_{j=w_{i-1}+1}^{w_{i}}\left[\left\|F\left(s(m)_{j}\right)-F\left(s(m)_{j-1}\right)\right\|\right. \\
& \left.\times 2 \sum_{k=w_{i-1}+1}^{w_{i}}\left\|F\left(s(m)_{k}\right)-F\left(s(m)_{k-1}\right)\right\|\left\|A^{q+2} p\right\|\right]
\end{aligned}
$$

(by (4.4), (4.3), and (4.1)).

Then, using the absolute continuity of $F$, the fact that $F$ is of bounded variation on 
$[u, v]$, and the fact that $s(1), s(2), \ldots$ is an admissible sequence of chains from $u$ to $v$, we have that $\lim _{n \rightarrow \infty} \prod_{s(n)}[A, F] A^{q} p$ exists. Hence, by (4.3) and the fact that $A$ is closed we see that $\lim _{n \rightarrow \infty} \prod_{s(n)}[A, F] p$ is in domain $A^{q}$ for every positive integer $q$ and so (2.6) is established. Thus, (IV) is satisfied and the theorem is proved.

We shall apply Theorem 4.1 to the following linear partial differential equation:

EXAMPLE 4.1. Let $X$ be the Banach space of continuous complex-valued functions defined on $(-\infty, \infty)$ and periodic with period $2 \pi$ and let $X$ have the supremum norm. Let $D$ be the set of members of $X$ having a continuous second derivative and define the operators $D_{0}, D_{1}, D_{2}$ on $D$ by $D_{0} x=-x, D_{1} x=x^{\prime}$, and $D_{2} x=x^{\prime \prime}$ for $x \in D$. Then, $D_{0}, D_{1}, D_{2}$ are closable linear operators on $D$ and $D$ is dense in $X$. In Kato [10] (Theorem 6) it is shown that if $p, q, r$ are real-valued $2 \pi$ periodic functions on $(-\infty, \infty)$ with continuous derivatives of second, first, and zero order, respectively, $p>0$ on $(-\infty, \infty), r \geqq 0$ on $(-\infty, \infty)$, and $p^{\prime \prime}-q^{\prime}-r \leqq 0$ on $(-\infty, \infty)$, then $p D_{2}+q D_{1}+r D_{0}$ has the following properties:

(4.5) $\left\|\left(I-\left(p D_{2}+q D_{1}+r D_{0}\right)\right) x\right\| \geqq\|x\|$ for $x \in D$,

(4.6) range $\left(I-\left(p D_{2}+q D_{1}+r D_{0}\right)\right)=X$.

Define $A=D_{2}+D_{1}+D_{0}$ with domain $D$. Then, $-A=I-\left(D_{2}+D_{1}\right)$ and so by (4.5) and (4.6) $A$ is one-to-one, onto and $A$ is closed on $D$. Further,

$$
E=\bigcap_{n=1}^{\infty} \text { domain } A^{n}=\left\{x \mid x^{(n)} \in X \text { for every positive integer } n\right\}
$$

Suppose that $f_{i}(t), i=0,1,2$, are real-valued functions on $[0, \infty)$ such that $f_{2}(t)$ is increasing and $f_{0}(t)$ is nondecreasing. Further, require that each of $f_{i}(t), i=0,1,2$, is absolutely continuous. If $t \geqq 0$ and $x \in X$ define $\left(F_{i}(t) x\right)(\xi)=f_{i}(t) x(\xi)$, $\xi \in(-\infty, \infty)$ and $i=0,1,2$, and we see that $F_{i}(t)$ is an absolutely continuous function from $[0, \infty)$ to $B(X)$. Define $A(t)=A$ for all $t \geqq 0$ and $F(t)=\sum_{i=0}^{2} F_{i}(t) D_{i} A^{-1}$ for all $t \geqq 0$. We see that if $0 \leqq s<t$ then $\|(I-(F(t)-F(s)) A) x\| \geqq\|x\|$ for $x$ in $D$ by (4.5) and range $(I-(F(t)-F(s)) A)=X$ by (4.6). Thus, (4.1) and (4.2) of Theorem 4.1 are satisfied. To show that (4.3) of Theorem 4.1 is satisfied we see that for $x \in E$ and $t>s \geqq 0$

$$
\begin{aligned}
A(F(t)-F(s)) A x & =\left(\sum_{i=0}^{2} D_{i}\right)\left(\sum_{j=0}^{2}\left(F_{j}(t)-F_{j}(s)\right) D_{j}\right) x \\
& =\sum_{i=0}^{2} \sum_{j=0}^{2}\left(F_{j}(t)-F_{j}(s)\right) D_{i} D_{j} x \\
& =\sum_{j=0}^{2} \sum_{i=0}^{2}\left(F_{j}(t)-F_{j}(s)\right) D_{j} D_{i} x \\
& =\left(\sum_{j=0}^{2}\left(F_{j}(t)-F_{j}(s)\right) D_{j}\right)\left(\sum_{i=0}^{2} D_{i}\right) x \\
& =((F(t)-F(s)) A) A x
\end{aligned}
$$


and so $A(I-(F(t)-F(s)) A) x=(I-(F(t)-F(s)) A) A x$. Then, for $x \in E$ and $t>s \geqq 0$ we have that $(I-(F(t)-F(s)) A)^{-1} x \in E$ and hence

$$
\begin{aligned}
(I-(F(t)-F(s)) A)^{-1} A x \\
=(I-(F(t)-F(s)) A)^{-1} A(I-(F(t)-F(s)) A)(I-(F(t)-F(s)) A)^{-1} x \\
=(I-(F(t)-F(s)) A)^{-1}(I-(F(t)-F(s)) A) A(I-(F(t)-F(s)) A)^{-1} x \\
=A(I-(F(t)-F(s)) A)^{-1} x
\end{aligned}
$$

and so (4.3) holds.

Then, we have by Theorem 4.1 that conditions (I), (II), (III), and (IV) are satisfied for $A, F$, and $E$ defined as above. Moreover, since $f_{i}(t), i=0,1,2$, is absolutely continuous, we have that for almost all $t$, if $x \in X$ and $\xi \in(-\infty, \infty)$, then

$$
\left(F^{\prime}(t) x\right)(\xi)=\sum_{i=0}^{2} f_{i}^{\prime}(t)\left(D_{i} A^{-1} x\right)(\xi)
$$

and so $F$ has a strong derivative almost everywhere on $[0, \infty)$. Thus, by Theorem 3.1 the evolution operator $U$ of $A$ with respect to $F$ exists on $\bar{E}=X$ and by Theorem 3.2 if $x \in E$ and $0 \leqq u$ then for almost all $t \geqq u$

$$
\delta^{+} / \delta t U(t, u) x=F^{+}(t) A U(t, u) x .
$$

Since the existence of the derivative in the norm of $X$ implies the existence of the derivative in the usual sense, we have established the following theorem:

THEOREM 4.2. Suppose that $f_{0}(t), f_{1}(t)$, and $f_{2}(t)$ are real-valued absolutely continuous functions on $[0, \infty)$ such that $f_{2}(t)$ is increasing and $f_{0}(t)$ is nondecreasing. Let $z$ be a continuous complex-valued function on $(-\infty, \infty), 2 \pi$ periodic, and infinitely differentiable on $(-\infty, \infty)$. If $s \geqq 0$ there is a complex-valued function $x(t, \xi)$ on $[s, \infty) \times(-\infty, \infty)$ such that $x$ is $2 \pi$ periodic and infinitely differentiable in $\xi, x(s, \xi)$ $=z(\xi)$ for all $\xi \in(-\infty, \infty)$, and for almost all $t \geqq s$

$$
\delta^{+} x(t, \xi) / \delta t=\sum_{i=0}^{2}\left(f_{i}^{\prime+}(t)\right) \delta^{i} x(t, \xi) / \delta \xi^{i}
$$

Moreover, $x(t, \xi)=(U(t, s) z)(\xi)$ for $t \geqq s$ and $\xi \in(-\infty, \infty)$, where $U$ is the evolution operator of $A$ with respect to $F$ on $E$ as in Example 4.1 above.

In order to apply Theorem 3.3 to this example we observe that for $D_{0}, D_{1}, D_{2}, A$, $F, E$ defined as above the constant $M(F, p, v)$ in (2.4) can be chosen as $\|A p\|$ for each $p \in E$ (by (4.3) and (2.3)). Suppose that $G$ is an absolutely continuous function from $[0, \infty)$ to $B(X)$ defined by $g_{i}(t), i=0,1,2$, in the same way that $F$ is defined by $f_{i}(t), i=0,1,2$. Let $U_{F}$ and $U_{G}$ be the evolution operators for $A$ with respect to $F$ and $G$, respectively; let $z \in E, s \geqq 0, x(t, \xi)=\left(U_{F}(t, s) z\right)(\xi)$, and $y(t, \xi)=$ 
$\left(U_{G}(t, s) z\right)(\xi)$ for $t \geqq s, \xi \in(-\infty, \infty)$. Then, for $\xi \in(-\infty, \infty)$ and almost all $t \geqq s$,

$$
\begin{array}{ll}
x(s, \xi)=z(\xi), & \delta^{+} x(t, \xi) / \delta t=\sum_{i=0}^{2}\left(f_{i}^{\prime+}(t)\right) \delta^{i} x(t, \xi) / \delta \xi^{i}, \\
y(s, \xi)=z(\xi), & \delta^{+} y(t, \xi) / \delta t=\sum_{i=0}^{2}\left(g_{i}^{\prime+}(t)\right) \delta^{i} y(t, \xi) / \delta \xi^{i}
\end{array}
$$

and by Theorem 3.3

$$
|x(t, \xi)-y(t, \xi)| \leqq\|A z\| \sum_{i=0}^{2}\left(\int_{s}^{t}\left|f_{i}^{\prime}(r)-g_{i}^{\prime}(r)\right| d r\right)\left\|D_{i} A^{-1}\right\| .
$$

We next give a nonlinear example in the Hilbert space. For recent results in the study of nonlinear dissipative operators in the Hilbert space the reader is referred to M. Crandall and A. Pazy [5], J. Dorroh [6] and [7], H. Brezis, M. Crandall, and A. Pazy [2], C. Pao and W. Vogt [17], and Y. Kōmura [12].

EXAmple 4.2. Let $X$ be a real Hilbert space and let $A$ be a (possibly nonlinear) operator from a subset $D$ of $X$ to $X$ such that $A$ is $m$-dissipative on $D$, i.e.,

(4.7) $\|(I-\lambda A) p-(I-\lambda A) q\| \geqq\|p-q\|$ for $\lambda>0, p, q \in D$,

(4.8) range $(I-\lambda A)=X$ for $\lambda>0$,

or, equivalently, $A$ is $m$-dissipative with respect to $F(t)=t I, t \geqq 0$ (Definition 2.2). We will require the following facts shown in [18, Lemma 1.2]:

(4.9) If $A$ is $m$-dissipative on $D, p \in D$, and $\left\{s_{i}\right\}_{i=0}^{n}$ is an increasing sequence of nonnegative numbers, then $\left\|\prod_{i=1}^{n}\left(I-\left(s_{i}-s_{i-1}\right) A\right)^{-1} p-p\right\| \leqq\left(s_{n}-s_{0}\right)\|A p\|$ and $\left\|A \prod_{i=1}^{n}\left(I-\left(s_{i}-s_{i-1}\right) A\right)^{-1} p\right\| \leqq\|A p\|$.

One can show that (4.7) and (4.8) imply that $A$ is maximal dissipative on its domain $D$, i.e., $A$ is maximal with respect to the following property:

(4.10) $(A p-A q, p-q) \leqq 0$ for all $p, q \in D$ (see, for example, M. Crandall and A. Pazy [5]).

We will also require the following facts shown in [5]:

(4.11) If $A$ is maximal dissipative on its domain $D,\left\{x_{n}\right\}_{n=1}^{\infty}$ is a sequence in $D$ converging to $z$, and $\left\{A x_{n}\right\}_{n=1}^{\infty}$ is bounded, then $z$ is in $D$.

(4.12) If $A$ is maximal dissipative on its domain $D,\left\{x_{n}\right\}_{n=1}^{\infty}$ is a sequence in $D$ converging to $z, z \in D$, and $\lim \sup _{n \rightarrow \infty}\left\|A x_{n}\right\| \leqq\|A z\|$, then $\left\{A x_{n}\right\}_{n=1}^{\infty}$ converges to $A z$.

Let $f$ be an increasing real-valued absolutely continuous function on $[0, \infty)$ and define $F(t)=f(t) I$ (where $I$ is the identity in $B(X)$ ), $A(t)=A$ for $t \geqq 0$, and $E=D$ $=$ domain $A$. It is easily seen that conditions (I), (II), and (III) are satisfied for $A, F$, and $E$. To show that condition (IV) is satisfied we first observe that (2.4) is satisfied by virtue of (4.9). We will prove that (2.5) holds by an indirect argument. Assume that there exists $p \in D, c>0$, and $t \geqq 0$ such that if $n$ is a positive integer there is a chain $s(n)$ from $t$ to $t+d_{n}$, where $0<d_{n}<1 / n$, and $\left\|A \prod_{s(n)}[A, F] p-A p\right\|$ $\geqq c$. By (4.9) we see that $\lim _{n \rightarrow \infty} \prod_{s(n)}[A, F] p=p$ and for each positive integer $n$ 
we see that $\left\|A \prod_{s(n)}[A, F] p\right\| \leqq\|A p\|$. Then, by (4.12) $\left\{A \prod_{s(n)}[A, F] p\right\}_{n=1}^{\infty}$ converges to $A p$ yielding a contradiction and hence (2.5) holds. To show that (2.6) holds we let $s(1), s(2), \ldots$ be a sequence of chains from $u$ to $v, 0 \leqq u \leqq v$, such that $\lim _{n \rightarrow \infty} \prod_{s(n)}[A, F] p$ exists. Then $\left\{A \prod_{s(n)}[A, F] p\right\}_{n=1}^{\infty}$ is bounded by (4.9) and so $\lim _{n \rightarrow \infty} \prod_{s(n)}[A, F] p \in D=E$ by (4.11). Hence, (2.6) holds and condition (IV) is satisfied. By Theorem 3.1 the evolution operator $U$ of $A$ with respect to $F$ exists on $\bar{E}$ and by Theorem 3.2 if $p \in E$ and $0 \leqq u$ then for almost all $t \geqq u$

$$
d^{+} / d t U(t, u) p=f^{\prime}(t) A U(t, u) p .
$$

In conclusion we give the following example in the case that $A$ is nonlinear, continuous, and everywhere defined:

Example 4.3. Let $X$ be a Banach space and let $A$ be a function from $[0, \infty)$ to $N(X)$ such that

(4.13) domain $A(t)=X$ for $t \geqq 0$.

(4.14) $A$ is continuous as a function from $[0, \infty) \times X$ to $X$ and $A$ is bounded on bounded subsets of $[0, \infty) \times X$.

(4.15) $A$ is $m$-dissipative with respect to $F(t)=t I \in B(X)$ for $t \geqq 0$.

Let $E=X$ and we see immediately that conditions (I), (II), and (III) are satisfied. We will establish the following fact in order to show that condition (IV) is satisfied:

(4.16) If $p \in X, 0 \leqq u \leqq v$, and $s=\left\{s_{i}\right\}_{i=0}^{m}$ is a chain from $u$ to $v$ then

$$
\begin{aligned}
\| \prod_{s} & {[A, F] p-p\left\|\leqq \sum_{i=1}^{m}\left(s_{i}-s_{i-1}\right)\right\| A\left(s_{i-1}\right) p \| . } \\
\left\|\prod_{i=1}^{m}\left[A, F, s_{i}\right] p-p\right\| & \leqq \sum_{i=1}^{m}\left\|\prod_{j=i}^{m}\left[A, F, s_{i}\right] p-\prod_{j=i+1}^{m}\left[A, F, s_{i}\right] p\right\| \\
& \leqq \sum_{i=1}^{m}\left\|\left[A, F, s_{i}\right] p-p\right\| \\
& =\sum_{i=1}^{m}\left\|\left[A, F, s_{i}\right] p-\left[A, F, s_{i}\right]\left(I-\left(s_{i}-s_{i-1}\right) A\left(s_{i-1}\right)\right) p\right\| \\
& \leqq \sum_{i=1}^{m}\left(s_{i}-s_{i-1}\right)\left\|A\left(s_{i-1}\right) p\right\| .
\end{aligned}
$$

We see then that (2.4) and (2.5) follow from (4.14) and (4.15), and furthermore, (2.6) is satisfied since $E=X$. Thus, conditions (I)-(IV) hold and we may apply Theorems 3.1 and 3.2. By Theorem 3.1 the evolution operator $U$ of $A$ with respect to $F(F(t)=t I, t \geqq 0)$ exists on $X$ and by Theorem 3.2 if $p \in X$ and $0 \leqq u$, then for $t \geqq u$

$$
d^{+} / d t U(t, u) p=A(t) U(t, u) p
$$

\section{REFERENCES}

1. F. E. Browder, Non-linear equations of evolution, Ann. of Math. (2) 80 (1964), 485-523. MR 30 \#4167.

2. H. Brezis, M. G. Crandall and A. Pazy, Perturbations of nonlinear maximal monotone sets in Banach space, Comm. Pure Appl. Math. 23 (1970), 123-153. 
3. H. Brezis and A. Pazy, Accretive sets and differential equations in Banach spaces (to appear).

4. M. G. Crandall and T. M. Liggett, Generation of semi-groups of nonlinear transformations on general Banach spaces (to appear).

5. M. G. Crandall and A. Pazy, Semi-groups of nonlinear contractions and dissipative sets, J. Functional Analysis 3 (1969), 376-418. MR 39 \#4705.

6. J. R. Dorroh, A nonlinear Hille-Yosida-Phillips theorem, J. Functional Analysis 3 (1969), 345-353. MR 39 \#2019.

7. - A class of nonlinear evolution equations in a Banach space, Trans. Amer. Math. Soc. 147 (1970), 65-74.

8: J. A. Goldstein, Abstract evolution equations, Trans. Amer. Math. Soc. 141 (1969), 159-185. MR 40 \#789.

9. E. Hille and R. S. Phillips, Functional analysis and semi-groups, rev. ed., Amer. Math. Soc. Colloq. Publ., vol. 31, Amer. Math. Soc., Providence, R. I., 1957. MR 19, 664.

10. T. Kato, Integration of the equation of evolution in a Banach space, J. Math. Soc. Japan 5 (1953), 208-234. MR 15, 437.

11. - Nonlinear semigroups and evolution equations, J. Math. Soc. Japan 19 (1967), 508-520. MR 37 \#1820.

12. Y. Kōmura, Nonlinear semi-groups in Hilbert space, J. Math. Soc. Japan 19 (1967), 493-507. MR 35 \#7176.

13. R. H. Martin, A global existence theorem for autonomous differential equation in a Banach space (to appear).

14. J. Mermin, Accretive operators and nonlinear semi-groups, Thesis, University of California, Berkeley, Calif., 1968.

15. J. W. Neuberger, Product integral formulae for nonlinear expansive semi-groups and nonexpansive evolution systems, J. Math. Mech. 19 (1969), 403-410.

16. S. Oharû and I. Miyadera, Approximation of semi-groups of nonlinear operators (to appear).

17. C. V. Pao and W. G. Vogt, On the stability of nonlinear operator differential equations, and applications (to appear).

18. G. F. Webb, Nonlinear evolution equations and product integration in Banach spaces, Trans. Amer. Math. Soc. 148 (1970), 273-282.

19. - Product integral representation of time dependent nonlinear evolution equations in Banach spaces, Pacific J. Math. 32 (1970), 269-281.

20. K. Yosida, Functional analysis, Die Grundlehren der math. Wissenschaften, Band 123, Academic Press, New York; Springer-Verlag, Berlin, 1965. MR 31 \#5054.

VANDERBILT UNIVERSITY, NASHVILLE, TENNESSEE 37203 